(7) H. W. Dodson and E. R. Hedeman, $A p . J$. xxo, 242, 1949.

(8) H. W. Newton, Jour. B.A.A. 57, 54, 1947.

(9) M. A. Ellison, The Observatory, 67, 206, 1947.

(10) M. A. Ellison and F. Hoyle, The Observatory, 67, 181, 1947.

(I I) L. Goldberg, H. W. Dodson and E. A. Müller, Ap. J. 120, 83, I954.

(r2) E. R. Mustel and A. B. Severny, Reports of Crim. Astrophys. Obs. 8, 19, 1952.

(r3) M. A. Ellison, Huitième Rapport de la Commission pour l'Etude des Relations entre les Phénomènes Solaires et Terrestres, p. 32 (Paris, 1954, Sennac).

(14) T. G. Cowling, The Sun (Kuiper), p. $5^{87}$.

\title{
DISCUSSION
}

H. AlFVÉn: Could you give some figures for the density of matter in the point where the flare starts?

M. A. Ellison: It would be very difficult to give a density figure for these active regions. The flare certainly begins at very different heights.

M. N. SAHA: I want to know whether observations have been made in other spectral lines, say those of $\mathrm{He}$ ?

M. A. ElLISON: We also observe very interesting effects in the $\mathrm{He} \mathrm{I}$ lines. The great need at present is to obtain simultaneous spectra in the Balmer series $(\mathrm{H} \alpha-\mathrm{H} \epsilon)$ of high dispersion and free from scattered light.

M. N. SAHA: Have lines in the Lyman series been observed during a flare?

M. Minnaert: No rocket observations have been made yet at such moments.

L. GOLDBERG: Is the dip in the red wing of $\mathrm{H} \alpha$ due to water-vapour absorption?

M. A. Ellison: Yes.

\section{THE FINE STRUCTURE OF FLARES}

\section{By A. B. SEverny}

Our new tower telescope has permitted us to discover some new peculiar characteristics of the facula- and flare-emission. The most striking feature is a concentration of the emission in very small grains, like smallpox, with a size of some hundred $\mathrm{km}$. The continuous emission looks like a very thin thread along the whole spectruin. The intensity of this thread increases steadily towards the violet. Also, the line emission appears as thin threads cutting the absorption lines. Generally, only the wings of the emission lines are observed, and no reversal appears in the core, as if the core were undisturbed. I would like to draw your attention to the interesting fact that sometimes only one of the wings (on the blue side) is observed. I have introduced the term 'moustaches' for this emission. Sometimes these moustaches can be followed up to several $\AA$ from the centre of the $\mathrm{H} \alpha$ line and up to Io $\AA$ from the centre of the $\mathrm{H}$ and $\mathrm{K}$ lines.

It should be emphasized that this fine structure has a short lifetime and can be observed only at perfect seeing conditions and with an instrument of good resolving power. It seems to us that these one-sided moustaches must be considered as observational evidence for the presence of corpuscular streams. This opinion is also based on results found by Dr Nikonov and myself from a photo-electric investigation of faculae and flares made in the spectral lines $H \alpha, H$ and $K$. Sometimes a very narrow and weak $K_{4}$ emission has been found in faculae and flares observed at the limb. If we consider an additional depression in the blue wing mentioned above as due to a cloud of $\mathrm{Ca}+$ particles ejected from the flare, the particle density will be $\mathrm{IO}^{4} \mathrm{~cm} \cdot{ }^{-3}$. The position of this depression corresponds to velocities of about $1000 \mathrm{~km}$. $/ \mathrm{sec}$.

According to our spectroscopic observations the continuous emission and the moustaches are pronounced in the very first stage of development of flares and faculae. The second and especially the post-maximum stage is characterized, as is well known, by a strong and broad line-emission which sometimes consists of thin threads too. 


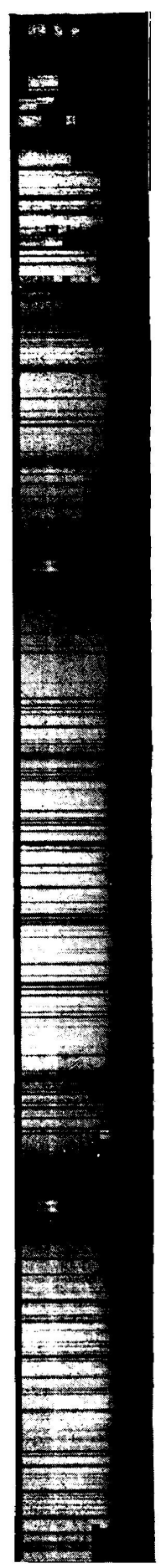

Fig. $\mathrm{I} a$

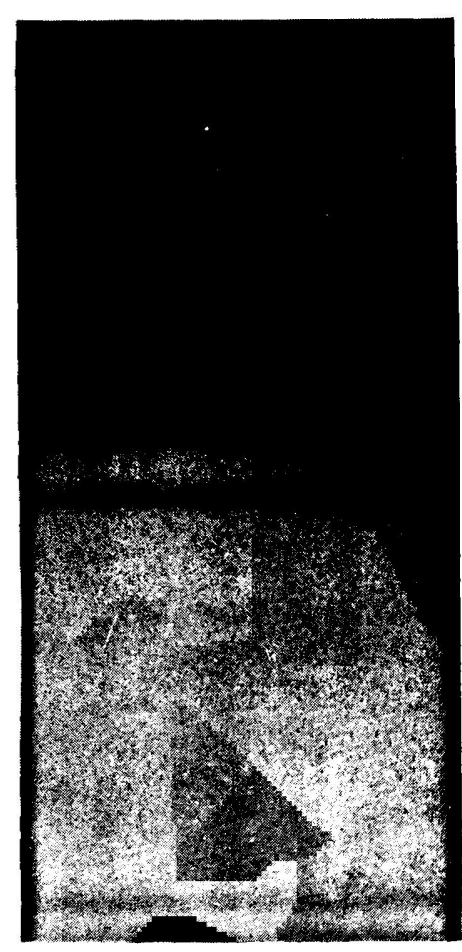

Fig. I $b$

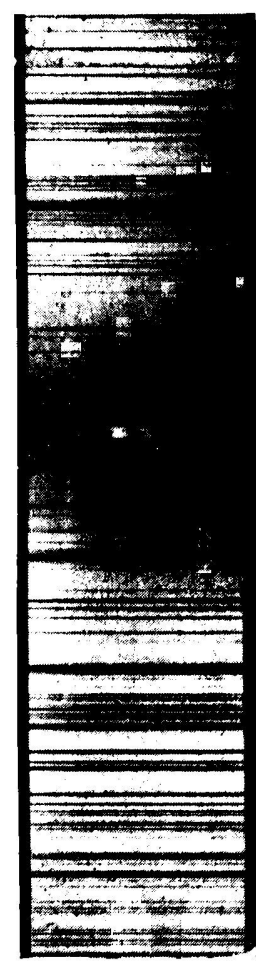

Fig. I $c$

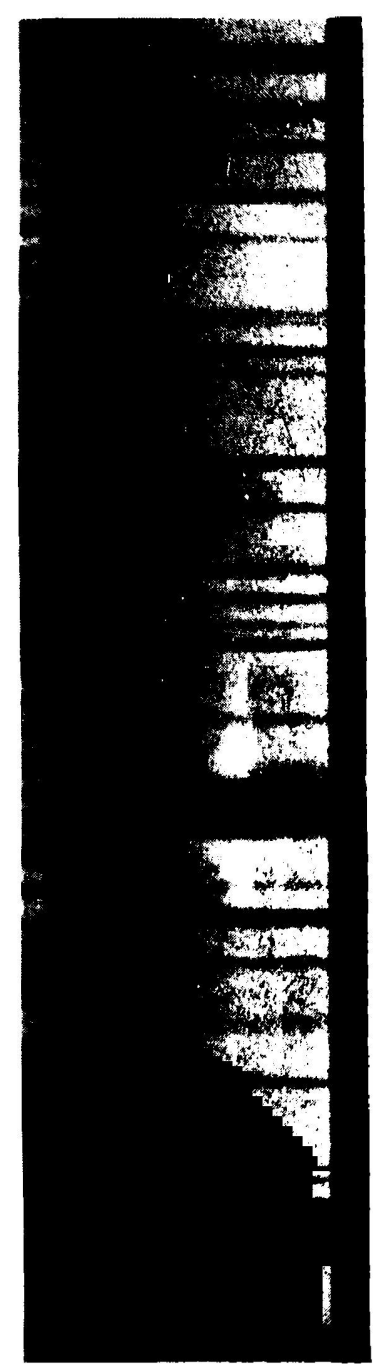

Fig. I $d$

Fig. I $a$. Continuous emission in the region $\mathrm{H}$ and $k$.

Fig. I $b$. One-sicled 'moustaches' $\mathrm{H} \alpha$-line.

Fig. I $c$. 'Moustaches' in K-line.

Fig. I $d$. 'Moustaches' in $\mathrm{H} \beta$-line. 
An analysis of the line-emission carried out by Mustel and myself has shown that a strong self-absorption takes place in flares. We were able to show in $\mathrm{I} 952$ that the $\mathrm{H} \alpha$ line is broadened by natural damping, whereas the other Balmer lines probably are broadened by the Stark effect. This can be seen from a comparison between theoretical and observed profiles. The data permit us to evaluate the flux of $L \alpha$ radiation and its selective pressure which appears to be sufficient for the ejection of hydrogen atoms with velocities up to $4000 \mathrm{~km}$. $/ \mathrm{sec}$.

A strong self-absorption takes place in the $\mathrm{H}$ and $\mathrm{K}$ lines too. The best agreement between theoretical and observed contours was obtained when assuming that the wings are broadened by natural damping and the central core by the Doppler effect (corresponding to $\mathrm{N}_{1}=5 \cdot 10^{15}, T_{\text {kin }}=5 \cdot 500$ and $v_{t}=0$ ). The minute to minute cinematography of flares and active regions shows the flare phenomenon to be an extremely dissipative process. For all the flares examined the increase in $\mathrm{H} \alpha$ line intensity is accompanied by a growth of the area. More than $30 \%$ of the flares show a marked and very rapid motion of luminous matter.

The larger the velocity of expansion of a flare, the higher is the maximum $\mathrm{H} \alpha$ intensity. The lifetime of a flare (halfwidth of light curve) increases as the square of its size. This is quite natural for any dissipative process. The surprisingly low optical depth $(0.8)$ in the centre of $\mathrm{H} \alpha$ for the limb flare of I6 August $\mathrm{I}_{952} 2$ showed that recombinations are not responsible for the observed emission.

The resolution of flares and faculae into small grains of emission, the peculiar character of continuous as well as line-emission and the characteristic features of their development lead to the conclusion that some intense explosive process, may be nuclear process, might be responsible for these phenomena. The small size of the grains indicates high density of energy and reveals the possibility of high-density electric currents, but does not exclude the nuclear processes too. The observed sharp edge of emission from one side and the diffuse edge from the other side favours the conception of a shock wave.

The abundance of such elements as $\mathrm{Li}, \mathrm{Be}$ and Deuterium in the solar atmosphere also favours the conception of nuclear explosions.

\section{CENTRE-LIMB EFFECTS IN SOLAR FLARES}

\section{By L. GoldberG}

It is well established that ionospheric disturbances in the Earth's atmosphere are caused by ultra-violet radiation associated with solar flares. Until recently, the correlation between the two phenomena was less than precise, since a certain number of bright flares were not accompanied by sudden ionospheric disturbances. Dodson (I955) has pointed out, however, that not all ionospheric disturbances are sudden, and has introduced the new designation of gradual ionospheric disturbance (G.I.D.) to denote the slowly increasing type of disturbance that frequently follows the onset of a flare. She has discovered further that when the product of maximum $\mathrm{H} \alpha$ intensity and total flare area exceeds a certain threshold value, an ionospheric disturbance, either sudden or gradual, will almost certainly take place.

The probability is also high that the $\mathrm{L} \alpha$ line is the ultra-violet radiation responsible for I.D.'s, since no other radiation of sufficient ionizing power appears capable of penetrating the atmosphere to the altitude of the D layer (Byram et al. I953; Goldberg, I954). If the $\mathrm{L} \alpha$ line is indeed responsible for D-layer disturbances, it becomes of great importance to know its intensity during solar flares. It is for this reason that strong attempts will be made to observe the line from high-altitude rockets during solar flares at the time of the International Geophysical Year. While it is likely that at least some of the attempts will prove successful, it would obviously be extremely fruitful to develop a successful theory for the flare-emitting layer which would permit the calculation of the L $\alpha$ intensity from observations of the Balmer series lines. 